\title{
Research on Multibeam Pattern Synthesis of Time Modulated Array Based on Convex Optimization
}

\author{
Jiawen Hu $\left(\mathbb{D}\right.$, Xikuan Dong $\mathbb{D}^{D}$, Jing Tan $(\mathbb{D}$, Yachao Jiang $\mathbb{D}$, and Hailin Li $\mathbb{D}$ \\ College of Electronic and Information Engineering, Nanjing University of Aeronautics and Astronautics, No. 29 Jiangjun Avenue, \\ Nanjing 210016, China
}

Correspondence should be addressed to Jiawen Hu; nuaahjw@nuaa.edu.cn

Received 10 January 2021; Revised 10 February 2021; Accepted 20 February 2021; Published 4 March 2021

Academic Editor: Giuseppe Castaldi

Copyright (c) 2021 Jiawen $\mathrm{Hu}$ et al. This is an open access article distributed under the Creative Commons Attribution License, which permits unrestricted use, distribution, and reproduction in any medium, provided the original work is properly cited.

An efficient multibeam pattern synthesis method for time modulated array (TMA) is proposed in this paper. The aim of this method is to generate a beam pattern on the specified sideband of TMA. The pattern synthesis problem is divided into two stages. In the first stage, the sequential convex optimization method is used to suppress the sidelobe of the center frequency and reduce the amplitude dynamic range ratios (DRR). In the second stage, the iterative convex optimization method is used to realize the multibeam pattern synthesis in the sideband. Owing to the introduction of convex optimization, the proposed method could be more efficient than approaches using a global optimization algorithm. The proposed method is capable of realizing pattern synthesis under different DRR constraints and different beam radiation directions. Numerical examples of two beams and four beams patterns of 1000-element time modulated linear array verify the effectiveness of the proposed method.

\section{Introduction}

Time modulated array (TMA) introduces a new degree of freedom of time on phased array [1]. Using a Radio Frequency (RF) switch to control the working state of each antenna array element, TMA can effectively reduce the complexity of the feed network, which makes it has the advantages of flexible application, high-performance, and multifunction [2]. With the modern communication system putting forward higher requirements for antenna array, more deep research has been done on TMA.

Due to the introduction of the RF switch, TMA generates sideband signals at each order of harmonics. For this reason, compared with the conventional array, the power loss of TMA also increases. Some research has derived the calculation of energy loss of TMA. The derivation of energy loss of uniform periodic time modulation array is given in [3]. In [4], nonuniform period time modulated arrays (NTMA) are investigated and analyzed in terms of radiated power. Therefore, the study of TMA must consider the influence of each order harmonic signal. Much research has been done in TMA to form an ideal pattern at the center frequency and suppressing sideband level [5-7]. Considering the independence of sidebands of TMA, sideband utilization of TMA has become a hot issue in recent years. Some research implements a new TMA system architecture to realize sideband beamforming. The multibeam pattern synthesis of the seventh order sideband of the 8-element linear array is realized by discrete Walsh transform in [8]. Gassab et al. [9] generated the Pen pattern in the first- and second-order sideband by using a SP3T based tripolar modulation signal. Multibeam synthesis of TMA composed of 8 subarrays is realized based on subarray division and multibranch structure in [10]. The second-order sideband multibeam formed of 20-element TMA is realized through weighted cosine and signal controlled time modulation in [11]. The sideband multibeamforming results of TMA under three different modulation signals are simulated in [12]. In [13], the dual-phase variable pulse amplitude (DPVPA) time sequence signal is designed to realize sideband beamforming. The periodic sum-of-weighted-cosine (SWC) pulses are proposed in [14] to realize sideband beamforming. The above research realizes sideband beamforming of TMA but also increases the complexity of system design. In some 
research, various optimization algorithms have been applied to optimize the static excitation and RF switch sequence and realize the multibeam pattern synthesis of TMA. The differential evolution algorithm (DE) is used to realize the sideband beamforming of time modulated linear array in [15] and is applied to the time modulated circular aperture array to obtain the first-order sideband beam pattern in [16]. The particle swarm optimization (PSO) algorithm is used to realize the beam pattern synthesis of the first three-order sidebands in [17]. Second-order sideband pencil beam pattern of TMA is synthesized by iterative FFT algorithm in [18]. The above research effectively solves the optimization problem of sideband beamforming of TMA through the global optimization algorithm. However, due to the characteristics of the global optimization algorithm, each optimization takes a long time and cannot ensure the optimality of each solution. Considering the optimization time, it is almost difficult for the global optimization algorithm to realize the optimization of large arrays (more than 1000 elements). The local optimal solution of the convex optimization problem is the global optimal solution. Turning the physical model into a convex optimization model can effectively reduce the optimization time and ensure the optimality of the solution [19]. Some research has applied convex optimization to the optimization of the ordinary array. By using the convex optimization algorithm to optimize the position and excitation of array elements, some research has effectively realized the pattern synthesis of the ordinary array. A novel synthesis method based on sequential convex optimization for nonuniformly spaced linear and planar arrays with frequency-invariant shaped patterns is proposed in [20]. A convex method for the synthesis of planar, maximally sparse, and steerable arrays is proposed in [21]. An effective method based on Bayesian compressive sensing (BCS) and convex optimization for near-field sparse array synthesis is presented in [22]. However, more variables need to be optimized, and the optimization objectives that need to be considered are more complex in TMA. The convex optimization method used in the ordinary array is no longer suitable for TMA. Therefore, how to combine convex optimization with multibeamforming of TMA is a topic worthy of exploration.

In this paper, an efficient multibeam pattern synthesis method is proposed. The convex optimization algorithm is applied to large-scale TMA that the past algorithm is difficult to apply. The optimization problem is divided into two parts: the sequential convex optimization algorithm is used to suppress sidelobe in the main frequency band and control the DRR in a low level. The iterative convex optimization algorithm is used to realize the sideband beamforming. The effectiveness of the proposed algorithm is verified in the case of DRR constraints and sideband radiation direction changes.

The structure of this paper is as follows: Section 2 introduces the target and optimization model of sideband multibeamforming for TMA. Section 3 introduces how to use the two-stage convex optimization algorithm to solve the optimization model. In Section 4, the effectiveness of this algorithm in sideband beamforming of TMA is verified by numerical simulation, and the influence of DRR constraints and sideband radiation direction changing on the optimization results is explored. Conclusions are drawn in Section 5.

\section{Mathematical Model}

As shown in Figure 1, without considering the coupling, the far-field radiation intensity of the uniform linear array is

$$
E(\theta, t)=e^{j 2 \pi f_{0} t} \sum_{n=1}^{N} \omega_{n} e^{j k(n-1) d \sin \theta},
$$

where $\theta$ is the radiation direction of the array, $f_{0}$ is the central frequency, $N$ is the number of elements, $I_{n}, \phi_{n}$ are the amplitude and phase excitation of the $n$-th element, $k=2 \pi / \lambda, \lambda$ is the wavelength of the incident wave, and $d$ is the spacing between adjacent elements.

Introduce the Switching function of the pulse shift timing scheme [23]:

$$
U_{n}(t)= \begin{cases}1, & t_{n} \leq t \leq t_{n}+\tau_{n} \\ 0, & \text { others. }\end{cases}
$$

As shown in Figure 2, $\tau_{n}, t_{n}$ are the switch-on duration time and switch-on time instant of the $n$-th element and $T_{P}$ is the modulation period of the switch. The far-field radiation intensity of time modulated linear array is obtained:

$$
E(\theta, t)=e^{j 2 \pi f_{0} t} \sum_{n=1}^{N} U_{n}(t) \omega_{n} e^{j k(n-1) d \sin \theta} .
$$

Expanding the switch function by Fourier transform,

$$
U_{n}(t)=\sum_{m=-\infty}^{+\infty} a_{m n} e^{j 2 \pi m f_{p} t},
$$

where $m=0, \pm 1, \pm 2, \pm 3 \ldots$, the radiation intensity of $m$-th sideband can be expressed as

$$
E_{m}(\theta, t)=e^{j 2 \pi\left(f_{0}+m f_{p}\right) t} \sum_{n=1}^{N} a_{m n} \omega_{n} e^{j k(n-1) \sin \theta},
$$

where $f_{p}=1 / T_{P}$ is the modulated frequency of the sequential switch and $a_{m n}$ is the Fourier coefficient of the $m$-th harmonic [24]:

$$
\begin{aligned}
a_{m n} & =\frac{1}{T_{P}} \int_{0}^{T_{P}} U_{n}(t) e^{-j 2 \pi m f_{p} t} d t \\
& =f_{p} \tau_{n} \sin c\left(m \pi f_{p} \tau_{n}\right) e^{-j m \pi f_{p}\left(2 t_{n}+\tau_{n}\right)} .
\end{aligned}
$$

The target of TMA multibeamforming is to achieve the following conditions when the radiation value of the central frequency band radiation direction is constant.

(1) The sidelobe level of the central frequency band is the minimum.

(2) The radiation level of the beamforming direction in the sideband is maximum.

(3) The radiation level of nonbeamforming direction in the sideband is minimum. 


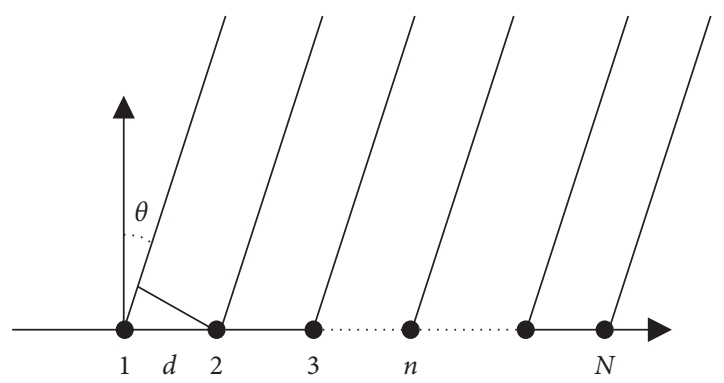

FIGURE 1: Uniform linear array.

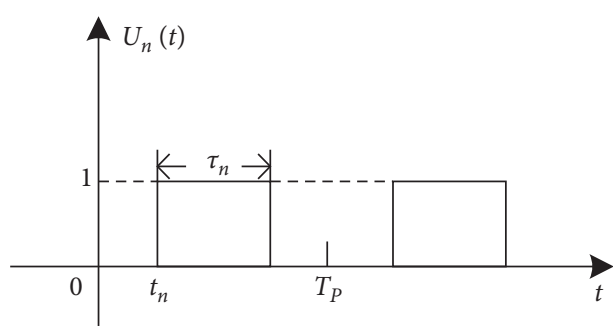

FIgURE 2: Switch function of RF switch.

The constraints on the center frequency band and sideband are shown in Figure 3, where the red line represents the center frequency pattern and the blue line represents the $m$-th sideband pattern. $\theta_{0}$ is the radiation direction on the central frequency. $\Omega_{l}$ is the sidelobe region on the central frequency. $\theta_{m}$ is the beamforming direction in the $m$-th sideband. $\Omega_{m l}$ represents the nonbeamforming direction area of the sideband. It can be expressed as the following combinatorial optimization problem model:

$$
\begin{aligned}
& \max \left|\mathbf{W}^{T} \mathbf{B}_{\theta_{l}} \mathbf{A}_{0}\right|, \quad \theta_{l} \in \Omega_{l}, \\
& \min _{\mathbf{W}, \mathbf{A}_{m}}\left|\mathbf{W}^{T} \mathbf{B}_{\theta_{m}} \mathbf{A}_{m}\right|, \quad m \neq 0, \\
& \max \left|\mathbf{W}^{T} \mathbf{B}_{\theta_{m l}} \mathbf{A}_{m}\right|, \quad m \neq 0, \theta_{m l} \in \Omega_{m l}, \\
& \text { s.t. } \mathbf{W}^{T} \mathbf{B}_{0} \mathbf{A}_{0}=1,
\end{aligned}
$$

where $\mathbf{W}=\left[\omega_{1}, \omega_{2}, \ldots, \omega_{N}\right]$ is the static excitation of the array, $(\bullet)^{T}$ is the conjugate transposition of the matrix, $\mathbf{B}_{\theta}=$ $\operatorname{diag}\left[1, e^{j k d \sin \theta}, \ldots, e^{j k(N-1) \sin \theta}\right]$ is the guide vector of the array, $\mathbf{A}_{m}=\left[a_{m 1}, a_{m 2}, \ldots, a_{m N}\right]^{T}, \mathbf{B}_{\theta_{l}}$ represents the guide vector of the sidelobe area of the central frequency, $\mathbf{B}_{\theta_{m}}$ represents the radiation direction guide vector of sideband, $\mathbf{B}_{\theta_{m l}}$ represents the guide vector of nonbeamforming direction of the sideband, and $\mathbf{B}_{0}$ represents the guide vector of the radiation direction of the main frequency.

Introducing the relaxation variable of radiation level constraint, the optimization problem in equation (7) is transformed into an inequality constrained optimization problem:

$$
\begin{aligned}
& \min _{\mathbf{W}, \mathbf{A}_{m}}-\left(\mathbf{W}^{T} \mathbf{B}_{0} \mathbf{A}_{0}\right), \\
& \text { s.t. }\left|\mathbf{W}^{T} \mathbf{B}_{\theta_{l}} \mathbf{A}_{0}\right| \leq u_{1}, \quad \theta_{l} \in \Omega_{l} \\
& -\left|\mathbf{W}^{T} \mathbf{B}_{\theta_{m}} \mathbf{A}_{m}\right| \leq u_{2}, \quad m \neq 0, \theta_{m} \in \Omega_{m} \\
& \left|\mathbf{W}^{T} \mathbf{B}_{\theta_{m l}} \mathbf{A}_{m l}\right| \leq u_{3}, \quad m \neq 0, \theta_{m l} \in \Omega_{m l}
\end{aligned}
$$

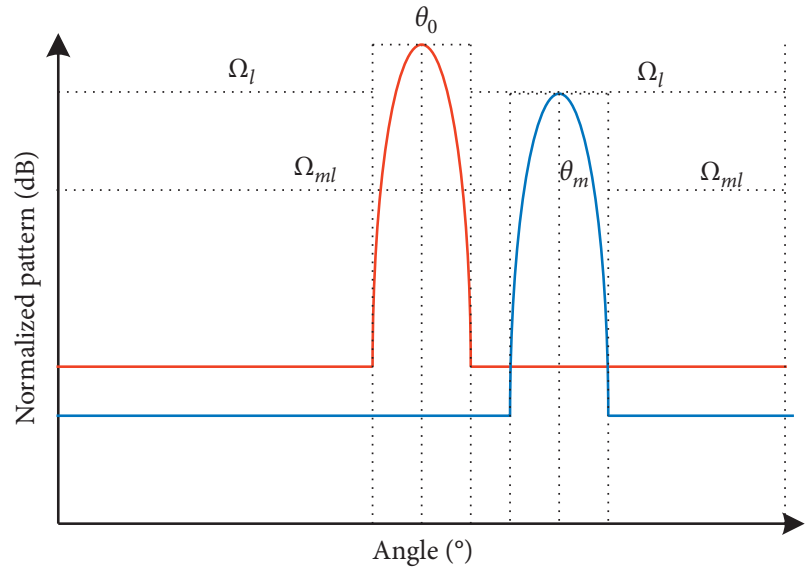

FIgURE 3: Constraint pattern.

where $u_{1}, u_{2}, u_{3}$ are the constraints of frequency band sidelobe level, sideband beamforming direction level, and sideband nonbeamforming direction level.

\section{Problem Solution}

The purpose of the optimization model in equation (8) is to realize the desired pattern in the center frequency and sideband of TMA by optimizing variables $\mathbf{A}_{m}$, W. Obviously, the optimization model in equation (8) is a highdimensional nonlinear problem and it is difficult to find the global optimal solution. Inspired by the method in [25], the problem in (8) is divided into two independent convex optimization problems and solved in two steps: the first constraint in (8) is solved in the first step and the second and third constraints are solved in the second step. Constraints are relaxed to reduce the nonconvexity in the optimization model:

(1) Obtain the beam pattern at the center frequency.

In the first step, $\mathbf{A}_{0}$, Ware optimized to obtain the beam pattern at the center frequency (the period of switching function is normalized to facilitate numerical simulation $\left.T_{P}=f_{P}=1, \tau_{n}, t_{n} \in[0,1]\right)$. The optimization model is shown as

$$
\begin{aligned}
\min _{\mathbf{W}, \mathbf{A}_{0}} u_{1}, & \\
\text { s.t. }\left|\mathbf{W}^{T} \mathbf{B}_{\theta_{l}} \mathbf{A}_{0}\right| & \leq u_{1} * \operatorname{Re}\left(\left|\mathbf{W}^{T} \mathbf{B}_{0} \mathbf{A}_{0}\right|\right), \quad \theta_{l} \in \Omega_{l}, \\
\left|\mathbf{A}_{0}\right| & \leq \mathbf{1}, \\
\operatorname{Im}\left(\mathbf{A}_{0}\right) & =0 \\
|\mathbf{W}| & \leq \mathbf{U}_{1}, \\
I_{0} \mathbf{U}_{1} & \leq \operatorname{Re}(\mathbf{W}),
\end{aligned}
$$

where $\mathbf{U}_{1}$ is the $n$-dimensional unit column vector and $I_{0}$ is the minimum amplitude excitation of array elements. After $\mathbf{A}_{0}, \mathbf{W}$ are obtained, according to equation (6), $a_{0 n}=\tau_{n}, \tau_{n}$ can be obtained directly from the optimization variables $\mathbf{A}_{0}$. 
(2) Obtain beam pattern in sideband.

In the second step, when $\mathbf{A}_{0}, \mathbf{W}$ are known, $t_{n}$ is optimized to obtain the beam pattern in the sideband. The optimization model is shown as

$$
\begin{aligned}
& \min _{t_{n}}-\sum\left[\operatorname{Re}\left(\mathbf{W}^{T} \mathbf{B}_{\theta_{m}} \mathbf{A}_{m}\right)\right], \quad m \neq 0, \theta_{m} \in \Omega_{m}, \\
& \text { s.t. }\left|\mathbf{W}^{T} \mathbf{B}_{\theta_{m l}} \mathbf{A}_{m l}\right| \leq u_{3}, \quad m \neq 0, \theta_{m l} \in \Omega_{m l} .
\end{aligned}
$$

Next, how to transform the optimization model in (9) and (10) into a convex model is introduced.

First, observe the optimization model in (9). Both W and $\mathbf{A}_{0}$ are optimization variables. Obviously, the optimization model is convex for the optimization variable $\mathbf{A}_{0}$ under a fixed $\mathbf{W}$ and is also convex for the optimization variable $\mathbf{W}$ under a fixed $\mathbf{A}_{0}$. So, the optimization model in (9) can be called the biconvex optimization model [26] for the optimization variables $\mathbf{A}_{0}, \mathbf{W}$. Inspired by the method in [27], alternating convex optimization (ACO) can be applied to solve the optimization model in (9). That is to solve a sequence of alternating weighted $l_{1}$ - norm optimization problems:

$$
\begin{aligned}
& \min _{\mathbf{A}_{0}} u_{1}, \\
& \text { s.t. }\left|\mathbf{W}_{*}^{T} \mathbf{B}_{\theta_{l}} \mathbf{A}_{0}\right| \leq u_{1} * \operatorname{Re}\left(\left|\mathbf{W}_{*}^{T} \mathbf{B}_{0} \mathbf{A}_{0}\right|\right), \quad \theta_{l} \in \Omega_{l}, \\
& \quad\left|\mathbf{A}_{0}\right| \leq \mathbf{1}, \\
& \operatorname{Im}\left(\mathbf{A}_{0}\right)=0 \\
& \min _{\mathbf{W}} u_{1}, \\
& \text { s.t. }\left|\mathbf{W}^{T} \mathbf{B}_{\theta_{l}} \mathbf{A}_{0}^{*}\right| \leq u_{1} * \operatorname{Re}\left(\left|\mathbf{W}^{T} \mathbf{B}_{0} \mathbf{A}_{0}^{*}\right|\right), \quad \theta_{l} \in \Omega_{l}, \\
& |\mathbf{W}| \leq \mathbf{U}_{1}, \\
& I_{0} \mathbf{U}_{1} \leq \operatorname{Re}(\mathbf{W}) .
\end{aligned}
$$

In problem (11a), $\mathbf{A}_{0}$ is the optimization variable under a given $\mathbf{W}_{*}$ that is obtained by solving problem (11b) in the previous step, and, in problem (11b), $\mathbf{W}$ is the optimization variable under a given $\mathbf{A}_{0}^{*}$, the solution to problem (6). Obviously, problems (11a) and (11b) are two convex optimization problems. In each iteration, problems (11a) and (11b) are solved sequentially. When the difference of $\mathbf{A}_{0}$ or $\mathbf{W}$ in two iterations is less than a certain value or reaches the maximum number of iterations, the iteration ends. In the first iteration, we can take $\mathbf{W}_{*}=\mathbf{U}_{1}$. Finally, $\mathbf{W}=\left[\omega_{1}, \omega_{2}, \ldots, \omega_{N}\right]$ is the element excitation and $\mathbf{A}_{0}=\left[\tau_{0}, \tau_{1}, \ldots, \tau_{N}\right]^{T}$ is the switch-ON duration time of the elements.

Next, after solving the optimization model in (11), the optimized static excitation $\omega_{n}$ and the switch-on duration time of the element $\tau_{n}$ are obtained. In order to transform the problem in (10) into a convex optimization model, the relationship between $\mathbf{W}^{T} \mathbf{B}_{\theta} \mathbf{A}_{m}$ and $t_{n}$ need to be further analyzed:

$$
\mathbf{W}^{T} \mathbf{B}_{\theta} \mathbf{A}_{m}=\sum_{n=1}^{N} \omega_{n} \tau_{n} \sin c\left(m \pi \tau_{n}\right) e^{-j m \pi\left(2 t_{n}+\tau_{n}\right)} e^{j k(n-1) \sin \theta} .
$$

For the optimization variable $t_{n}$, the nonconvexity in equation (12) is generated by $e^{-j 2 m \pi t_{n}}$. Inspired by the method in [28], an iterative convex optimization method can effectively solve this problem:

In the $i$-th iteration, make

$$
2 t_{n}^{i}=2 t_{n}^{i-1}+\delta_{n}^{i}
$$

where $a$ is a real increment close to zero. By the approximation of complex exponential function, there is

$$
e^{j x} \stackrel{x}{\longrightarrow} \longrightarrow 01+j x
$$

So, in the $i$-th iteration, there is

$$
e^{-j m \pi\left(2 t_{n}^{i}+\tau_{n}\right)}=\left(1-j m \pi \delta_{n}^{i}\right) e^{-j m \pi\left(2 t_{n}^{i-1}+\tau_{n}\right)} .
$$

Synthesize equations (12) and (15), there is

$$
\begin{aligned}
\left(\mathbf{W}^{T} \mathbf{B}_{\theta} \mathbf{A}_{m}\right)^{i} \approx & \sum_{n=1}^{N} \omega_{n} \tau_{n} \sin c\left(m \pi \tau_{n}\right)\left(1-j m \pi \delta_{n}^{i}\right) \\
& \cdot e^{-j m \pi\left(2 t_{n}^{i-1}+\tau_{n}\right)} e^{j k(n-1) \sin \theta}
\end{aligned}
$$

where $\omega_{n}, \tau_{n}$ can be regarded as known quantities, $t_{n}^{i-1}$ is the optimization result $t_{\mathrm{n}}$ in the last iteration. So, in the $i$-th iteration, $\delta_{n}^{i}$ is the only optimization variable. Take $\delta^{i}=$ $\left[\delta_{1}^{i}, \delta_{2}^{i}, \ldots, \delta_{N}^{i}\right]^{T}$ and $\mathbf{D}_{m}^{i-1}=\left[\omega_{1} \tau_{1} \sin c\left(m \pi \tau_{1}\right)\right.$ $\left.e^{-j m \pi\left(2 t_{1}^{i-1}+\tau_{1}\right)}, \ldots, \omega_{N} \tau_{N} \sin c\left(m \pi \tau_{N}\right) e^{-j m \pi\left(2 t_{N}^{i-1}+\tau_{N}\right)}\right]^{T}$. Convert equation (16) to matrix form:

$$
\left(\mathbf{W}^{T} \mathbf{B}_{\theta} \mathbf{A}_{m}\right)^{i}=\left(\mathbf{U}_{1}-\mathrm{jm} \pi \boldsymbol{\delta}^{i}\right)^{T} \mathbf{B}_{\theta} \mathbf{D}_{m}^{i-1}
$$

Then, the convex optimization form of the optimization model (10) in the $i$-th iteration is obtained

$$
\begin{aligned}
& \min _{\boldsymbol{\delta}_{m}^{i}}-\sum\left[\operatorname{Re}\left(\left(\mathbf{1}-\mathrm{jm} \pi \boldsymbol{\delta}^{i}\right)^{T} \mathbf{B}_{m} \mathbf{D}_{m}^{i-1}\right)\right], \quad m \neq 0, \theta_{m} \in \Omega_{m}, \\
& \text { s.t. }\left|\left(\mathbf{1}-\mathrm{jm} \pi \boldsymbol{\delta}^{i}\right)^{T} \mathbf{B}_{m l} \mathbf{D}_{m}^{i-1}\right| \leq u_{3}, \quad m \neq 0, \theta_{m l} \in \Omega_{m l}, \\
& \left|\delta_{n}^{i}\right| \leq \mu,
\end{aligned}
$$

where $\mu$ is the constraint for increment $\delta_{n}^{i},\left|\delta_{n}^{i}\right| \leq \mu, \mu=$ $(1 / 2 \pi) \approx 0.16$ [28]. Obviously (18) is a convex optimization model about $\delta^{i}$. When the difference in $\delta^{i}$ between two iterations is less than a certain value or reaches the maximum number of iterations, the iteration ends. $t_{n}$ can be solved by equation (13).

The variables $\omega_{n}, \tau_{n}$ are constrained in equation (11), which can effectively control the value range of array element static amplitude excitation $I_{n}$ and reduce the amplitude dynamic range ratios (DRR). At the same time, the optimization variables of the sideband optimization process are reduced, which could reduce the number of iterations and optimization time of the iterative convex optimization.

The main steps of multibeamforming of TMA based on convex optimization are as follows, and the flow chart is shown in Figure 4.

(1) Array Initialization. Set the number of array elements, array element spacing, main lobe width of 
center frequency band, sideband beamwidth, angle sampling interval, and so forth. The maximum number of iterations $Q$, parameter $u_{3}$, and sideband order $m$ are set.

(2) According to the optimization model in (11), set $i=1, \mathbf{W}_{*}^{(i)}=\mathbf{U}_{1}$, solve the convex problem in (11a), and get $\mathbf{A}_{0}$. Update $\mathbf{A}_{0}^{*(i)}=\mathbf{A}_{0}$, solve the convex problem in (11a), and get $\mathbf{W}, \mathbf{W}_{*}^{(i+1)}=\mathbf{W}$.

(3) If the end condition is reached, iteration ends. The optimized variables $\omega_{n}, \tau_{n}$ are obtained by $\mathbf{W}, \mathbf{A}_{0}$. If not, return to (2) and repeat iteration.

(4) Under the condition that $\omega_{n}, \tau_{n}$ are known, set $i=1, t_{n}^{(i)}=0$, calculate $\mathbf{D}_{n}^{(i)}$, solve the problem in (18), get $\delta^{i}$, and calculate $t_{n}, t_{n}^{(i+1)}=t_{n}$.

(5) If the end condition is reached, iteration ends. The optimized $t_{n}$ is obtained. If not, return to (4) and repeat iteration.

(6) Display the final optimized radiation pattern.

\section{Simulation Results}

In order to verify the performance of the proposed algorithm in the beamforming of time modulated array, four experiments are designed in 1000-element time modulated linear array.

(1) The effect verification of single sideband beamforming for large time modulated array based on two-stage convex optimization.

(2) The influence analysis of two-stage convex optimization on single sideband beamforming of large time modulated array with different DRR constraints.

(3) The influence analysis of different sideband beamforming directions on the single sideband beamforming of large time modulated array.

(4) The effectiveness verification of multisideband beamforming method for large time modulated array based on two-stage convex optimization.

\subsection{The Effect Verification of Single Sideband Beamforming for} Large Time Modulated Array Based on Two-Stage Convex Optimization. In order to verify the effectiveness of the sideband beamforming method for TMA based on two-stage convex optimization, the algorithm is used to generate a beam pattern in the specified direction of the first sideband. The array parameters and pattern parameters are shown in Table 1.

$N$ is the number of array elements, $d$ is the spacing between adjacent array elements, $\theta_{0}, \theta_{1}$ are the radiation direction of the center frequency and the beamforming direction of the first sideband, $F N B W_{0}, F N B W_{1}$ are the pencil beamwidth of the main frequency band and the first sideband, respectively, $u_{3}$ is the nonbeam direction constraint of the sideband, and $I_{0}$ is the minimum static excitation amplitude constraint $\left(I_{0}=1 / D R R\right)$.

Using the method proposed in this paper, the constrained pattern is obtained in 1971.44 seconds. The effect of the pattern on the center frequency and the first two

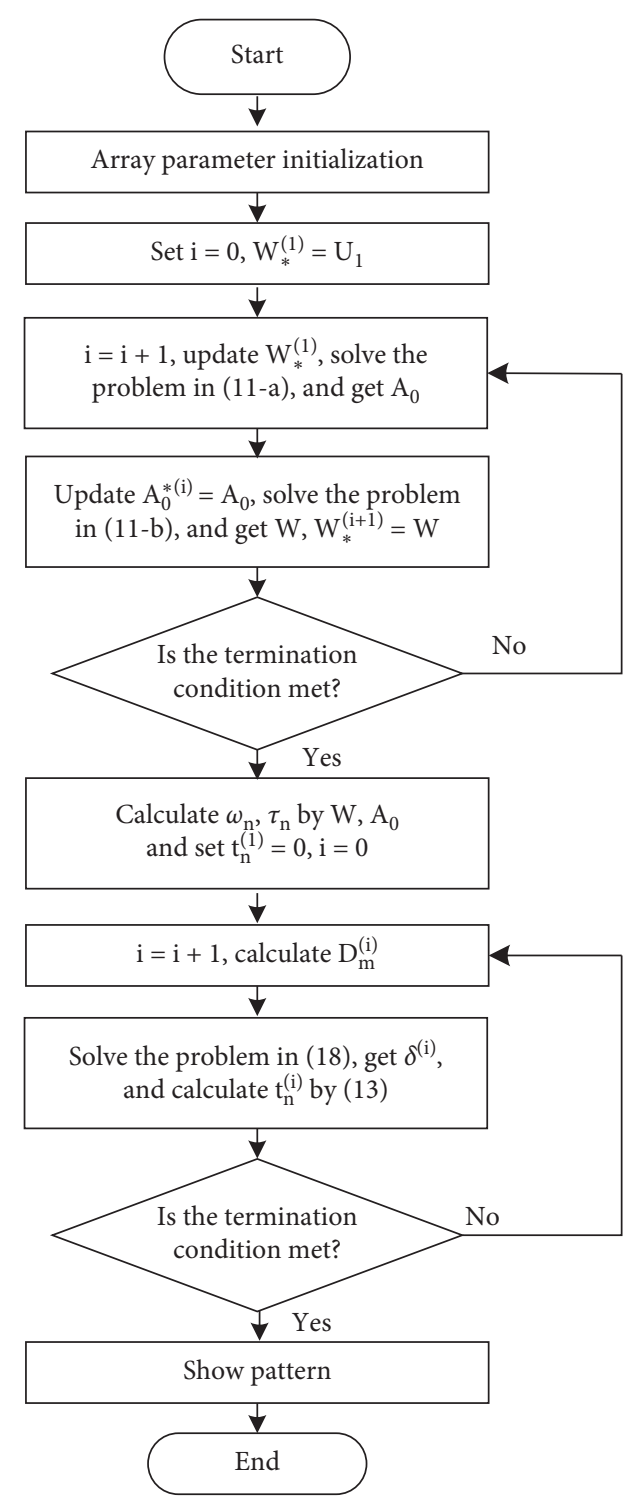

FIGURE 4: Flow chart of the proposed algorithm.

sidebands is shown in Figure 5. Figure 6 shows the maximum sideband level from the first sideband to the $15^{\text {th }}$-order sideband after optimization. It can be seen from the two figures that the maximum sidelobe level (SLL) of the optimized main frequency band is $-35 \mathrm{~dB}$, the first sideband beam direction level (SBL1) is $-13.9 \mathrm{~dB}$, the maximum nonbeam direction level is $-36.2 \mathrm{~dB}$, the maximum relative level of the first sideband beam direction and nonbeam direction (SLL1) is $-22.3 \mathrm{~dB}$, and the maximum level of the $2^{\text {nd }}-15^{\text {th }}$ sideband (SBL2-SBL15) is $-32.3 \mathrm{~dB}$.

It can be seen from the results in two figures that the beam pattern is obtained at the desired frequency (center frequency and first sideband) after optimization with the proposed method. At the same time, the radiation intensity of undesired frequency (the second- and higher-order sideband) is obviously suppressed.

Figure 7 shows the normalized excitation amplitude distribution obtained by the sequential convex optimization 
TABLE 1: Array parameter.

\begin{tabular}{lc}
\hline Parameter name & Parameter value \\
\hline$N$ & 1000 \\
$d$ & $\lambda / 2$ \\
$\theta_{0}$ & $0^{\circ}$ \\
$\theta_{1} W_{0}$ & $15^{\circ}$ \\
$F N B W_{0}$ & $0.4^{\circ}$ \\
$F N B W_{1}$ & $1^{\circ}$ \\
$u_{3}$ & $-30 \mathrm{~dB}$ \\
$I_{0}(D R R)$ & $0.5(2)$ \\
\hline
\end{tabular}

algorithm. It can be seen from the figure that the normalized static excitation amplitude of 808 elements is greater than 0.95 , the minimum normalized excitation amplitude of the remaining 192 elements is 0.52 , and the system DRR is 1.92 , which meets the given constraint conditions.

In order to verify the convergence effect of convex optimization in the two steps, the convergence curves of the two steps are shown in Figure 8. Figure 8(a) shows the relationship between the number of iterations and SLL in the first stage. Figure 8(b) shows the relationship between the number of iterations and SBL1 in the second stage. It can be seen from the figure that the iterations of the two stages converge at the $3^{\text {rd }}$ and $6^{\text {th }}$ iterations, respectively.

In order to verify the optimization effect of the algorithm, changing the first sideband constraint of the optimization model in [25], the beam can be generated in the first sideband, setting the same simulation environment and the same array parameter as Table 1. The optimization results of the algorithm proposed in this paper are compared with those of the convex algorithm proposed in [25]. The results are shown in Table 2.

It can be seen from the results in Table 2 that, compared with the algorithm in [25], the proposed algorithm costs more optimization time, but from the results in [25], it takes thousands of hours to get the optimization results by using the global optimization algorithm.

Compared with the optimization results in [25], the optimization results of the proposed algorithm reduce the SLL $5 \mathrm{~dB}$, SBL is reduced by $5.3 \mathrm{~dB}$, SLL1 is reduced by $8.4 \mathrm{~dB}$, and energy loss is reduced by $0.08 \%$ [29]. The obtained solution is more advantageous. It is proved that the proposed algorithm is effective in realizing the main frequency band sidelobe suppression and single sideband beamforming optimization of time modulated array.

\subsection{The Influence Analysis of Two-Stage Convex Optimization} on Single Sideband Beamforming of Large Time Modulated Array with Different DRR Constraints. Simulation 1 verifies the effectiveness of the proposed algorithm. In order to further verify the optimization effect of the proposed algorithm under different DRR constraints, the minimum static excitation amplitude constraint $I_{0}$ in the first stage is changed. The main frequency band and sideband are optimized under other conditions, the same as Table 1. Figure 9 shows the distribution of excitation amplitude after two-stage convex optimization under different DRR constraints, and Table 3 shows the comparison results.

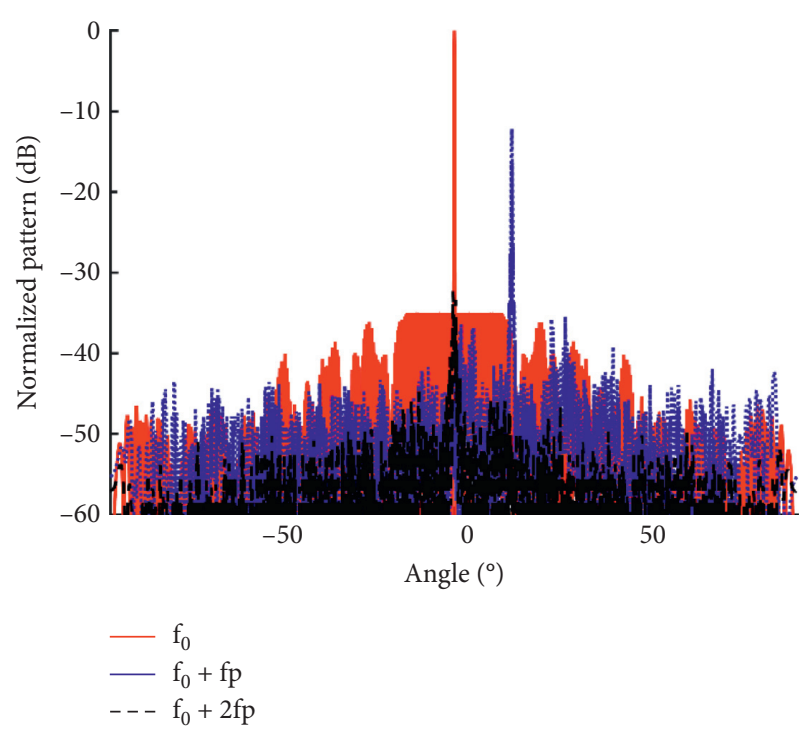

FIGURE 5: Optimized pattern at the central frequency and at the first two sidebands of TMA.

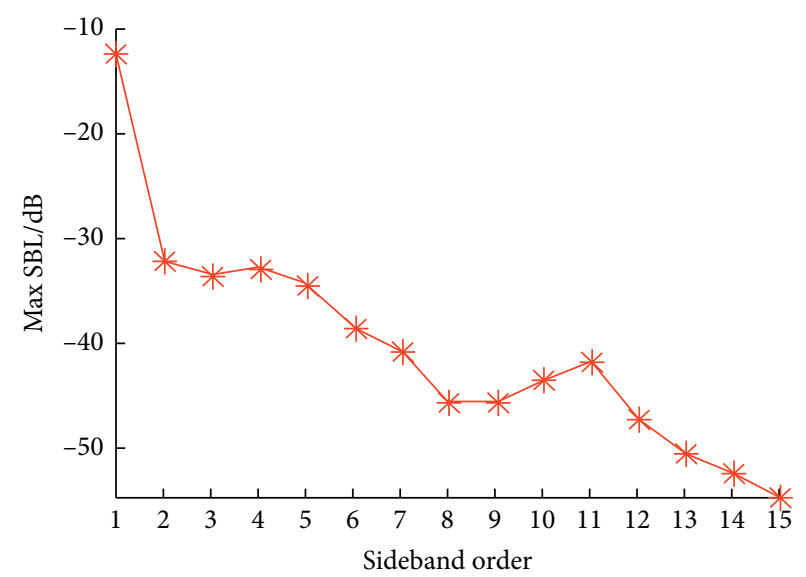

Figure 6: The SBL from the first sideband to the $15^{\text {th }}$ sideband.

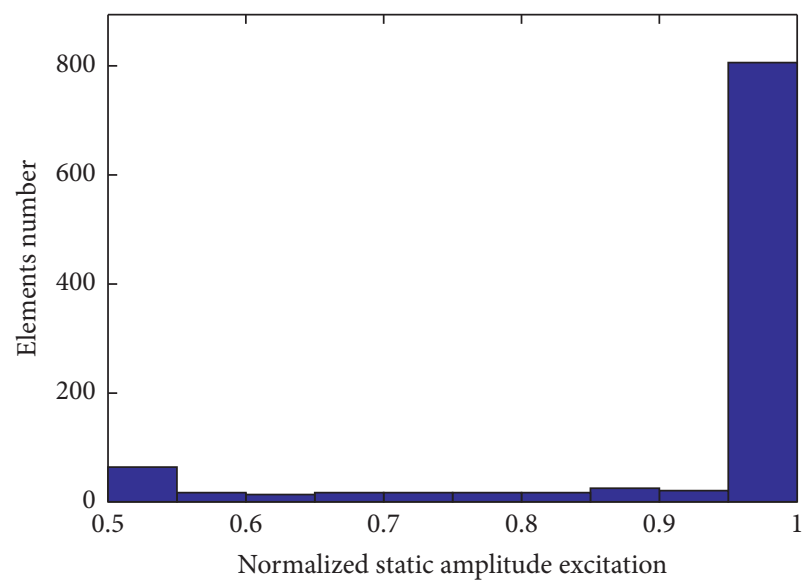

Figure 7: Normalized static amplitude excitation distribution.

$\mathrm{N} 1$ is the number of iterations when the sequential convex optimization converges, and N2 is the number of iterations when the iterative convex optimization converges. 


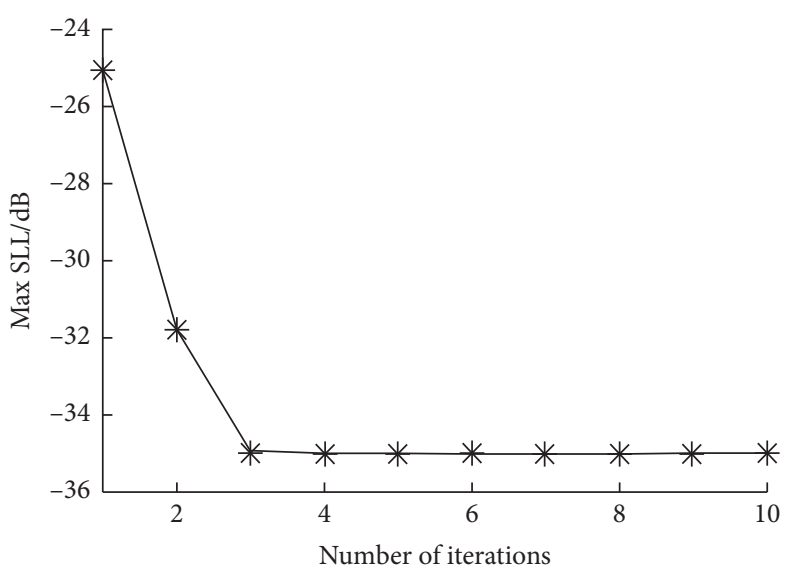

(a)

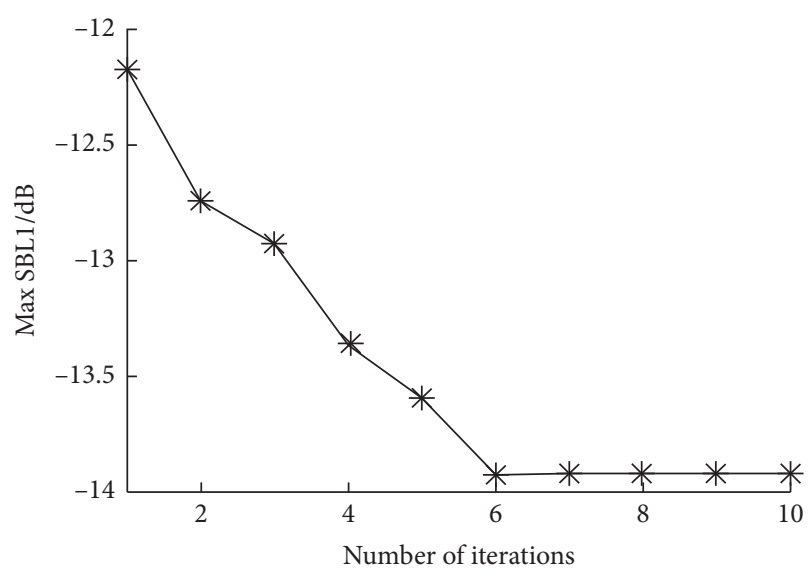

(b)

Figure 8: Convergence curve of two stages: (a) the first stage and (b) the second stage.

TABle 2: Comparison of the optimized results between the proposed method and the method in [25].

\begin{tabular}{lccccc}
\hline & SLL $(\mathrm{dB})$ & SBL $(\mathrm{dB})$ & SLL1 $(\mathrm{dB})$ & Power loss $(\%)$ & Opt. time $(\mathrm{s})$ \\
\hline Prop. & $-\mathbf{3 5}$ & $-\mathbf{3 2 . 3}$ & $\mathbf{- 2 2 . 3}$ & $\mathbf{0 . 5 2}$ & 1971.44 \\
Method in [22] & -30 & -27.0 & -15.1 & 0.6 & 360.64 \\
\hline
\end{tabular}

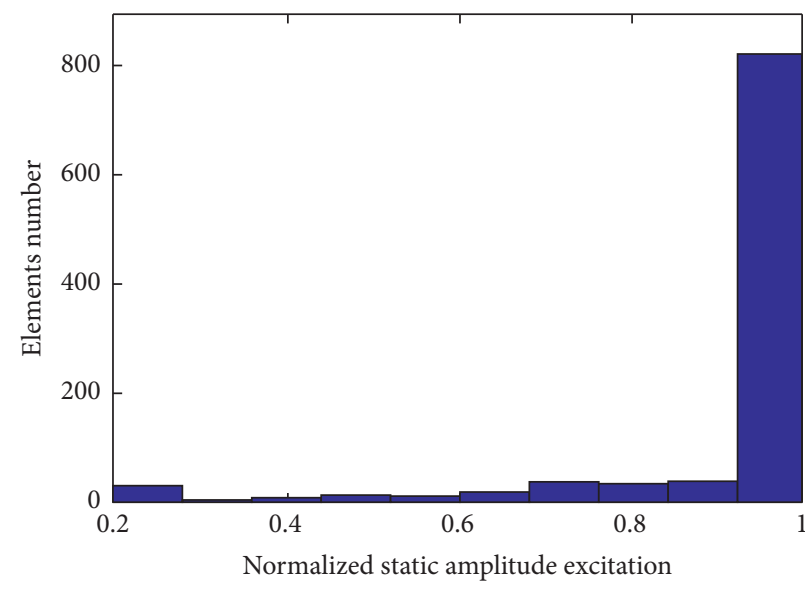

(a)

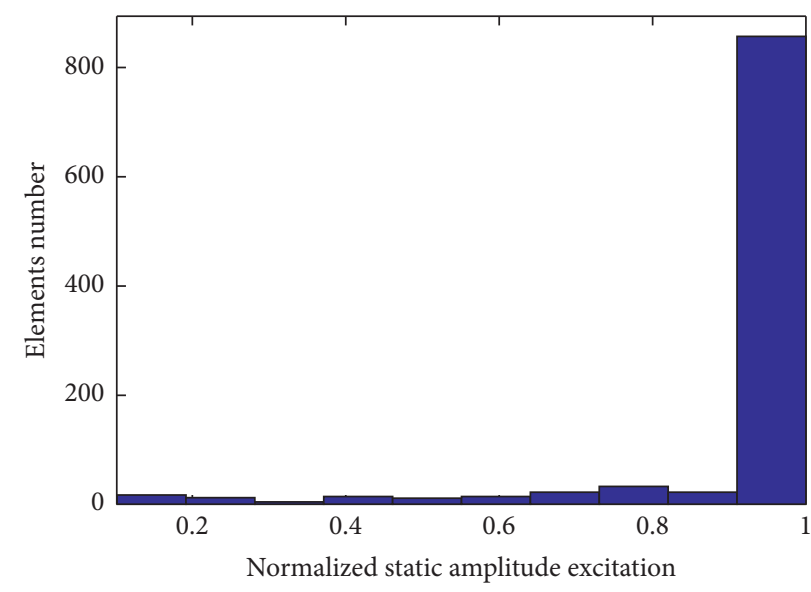

(b)

FIGURE 9: Normalized static amplitude excitation distribution with different DRR. (a) DRR = 5 and (b) DRR = 10 .

The results of Figure 8 show that when the constraint maximum DRR is 5 , the normalized static excitation amplitude of 824 elements is greater than 0.95 , the minimum normalized excitation amplitude of the remaining 176 elements is 0.22 , and the system DRR is about 4.55 and when the constraint maximum DRR is 10 , the normalized static excitation amplitude of 861 elements is greater than 0.95 , the minimum normalized excitation amplitude of the remaining 139 elements is 0.12 , and the system DRR is about 8.3.

The results in Table 3 show that, under different DRR constraints, the desired pattern can be obtained by using the proposed method. The maximum SLL in the main frequency band is $-35 \mathrm{~dB}$, and SBL1 and SBL2 in the sideband meet the constraint $u_{3}$. The maximum SLL1 is $-23.3 \mathrm{~dB}$, and the minimum SLL1 is $-25.5 \mathrm{~dB}$. When the DRR constraint is relaxed, the number of iterations and optimization time of the sequential convex optimization algorithm will increase, but the convergence can be achieved at the fourth time at most. The effectiveness of the two-stage convex optimization for large time modulated array single sideband beamforming with different DRR constraints is proved.

\subsection{The Influence Analysis of Different Sideband Beamforming Directions on the Single Sideband Beamforming of Large Time} Modulated Array. In order to verify the beam scanning 
TABLe 3: Comparison of optimization results under different DRR constraints.

\begin{tabular}{lccc}
\hline DRR & 2 & 5 & 10 \\
\hline Max SLL (dB) & -35 & -36 & -38 \\
Max SBL (dB) & -32.3 & -34.1 & -35.2 \\
Max SLL1 (dB) & -22.3 & -23.1 & -23.5 \\
N1 & 3 & 3 & 4 \\
N2 & 6 & 6 & 6 \\
Opt. time (s) & 1971.44 & 2042.65 & 2452.65 \\
\hline
\end{tabular}

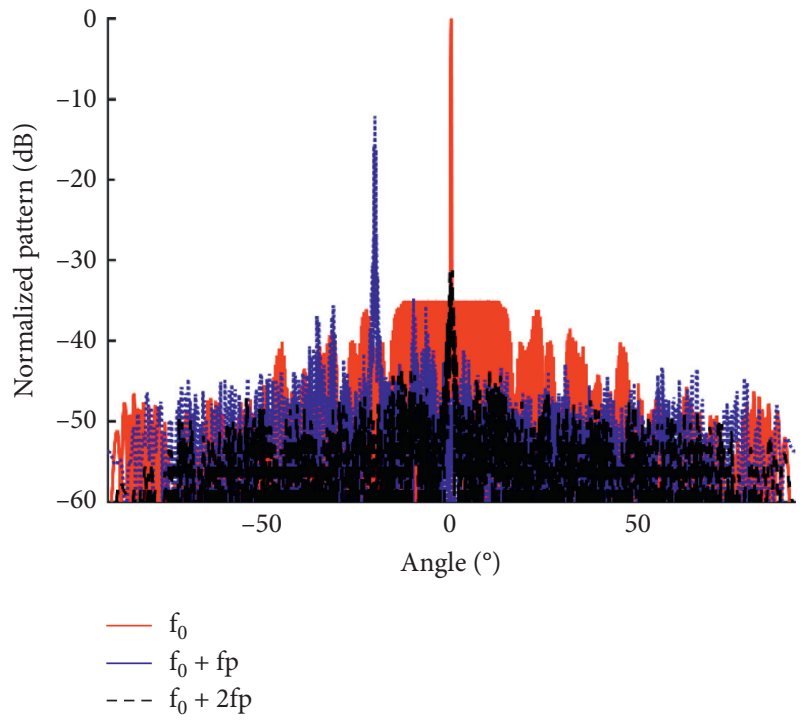

(a)
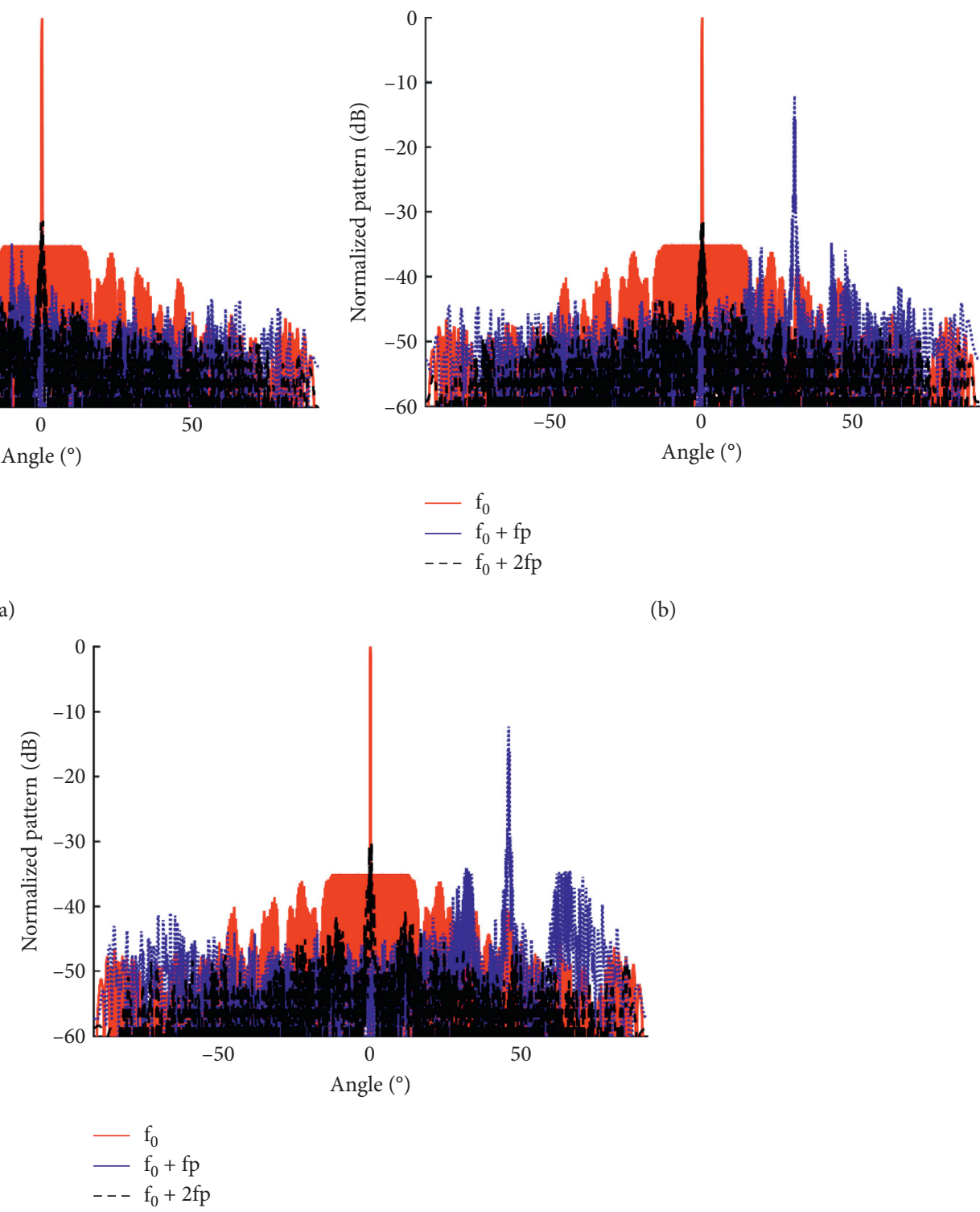


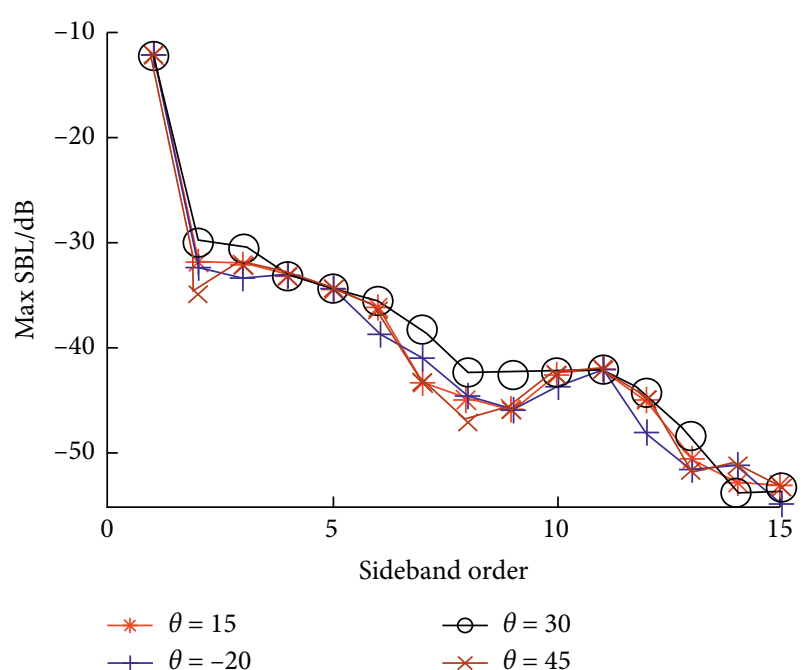

FIGURE 11: Max SBL from the second sideband to the $15^{\text {th }}$ sideband of different beamforming directions.

TABLE 4: Comparison of optimization results under different $\theta_{1}$.

\begin{tabular}{lcccc}
\hline$\theta_{1}\left(^{\circ}\right)$ & 15 & -20 & 30 & 45 \\
\hline Max SLL $(\mathrm{dB})$ & -35 & -35 & -35 & -35 \\
Max SBL $(\mathrm{dB})$ & -32.7 & -32.3 & -32.8 & -31.6 \\
Max SLL1 $(\mathrm{dB})$ & -22.5 & -22.3 & -22.7 & -21.9 \\
\hline
\end{tabular}

algorithm. The contrast effect of optimized patterns with different beamforming directions is shown in Figure 10. Figure 11 shows the maximum sideband levels from the second sideband to the $15^{\text {th }}$ sideband after optimization of different beamforming directions. Detailed comparative data are given in Table 4.

The results of Figures 10 and 11 and Table 4 show that the desired pattern can be obtained by changing the beamforming direction of the first sideband. The sidelobe level of the center frequency is almost unchanged. After changing the radiation direction of the first sideband to $-20^{\circ}, 30^{\circ}, 45^{\circ}$, respectively, the SBL is changed to $-32.3 \mathrm{~dB},-32.8 \mathrm{~dB}$, and $-31.6 \mathrm{~dB}$ and the SLL1 is changed to $-22.3 \mathrm{~dB},-22.7 \mathrm{~dB}$, and $-21.9 \mathrm{~dB}$. At the same time, the radiation intensity of the second- and higher-order sidebands is less than $-30 \mathrm{~dB}$. The constraints given in (8) are satisfied.

4.4. The Effectiveness Verification of Multisideband Beamforming Method for Large Time Modulated Array Based on Two-Stage Convex Optimization. 4.1 verifies the effectiveness of the proposed algorithm in single sideband beamforming. In order to further meet the needs of multiple beams in practical application, the proposed algorithm is applied to high-order sideband beamforming while keeping other conditions the same as Table 1 . The effect of the pattern on the main frequency band and the first three-order sidebands is shown in Figure 11, where the second sideband beam radiation direction $\theta_{2}=-20^{\circ}$, beamwidth $F N B W 2=2^{\circ}$, the third sideband beam radiation direction $\theta_{3}=30^{\circ}$, and beamwidth $F N B W 3=2^{\circ}$. Figure 12 shows the maximum sideband level from the first sideband to

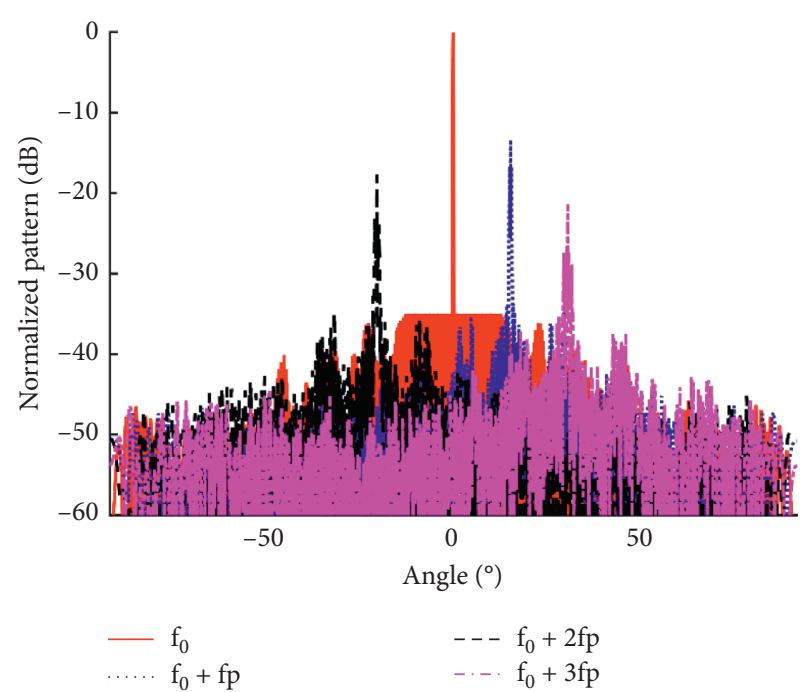

FIGURE 12: Optimized pattern at the center frequency and at the front three sidebands of time modulated array.

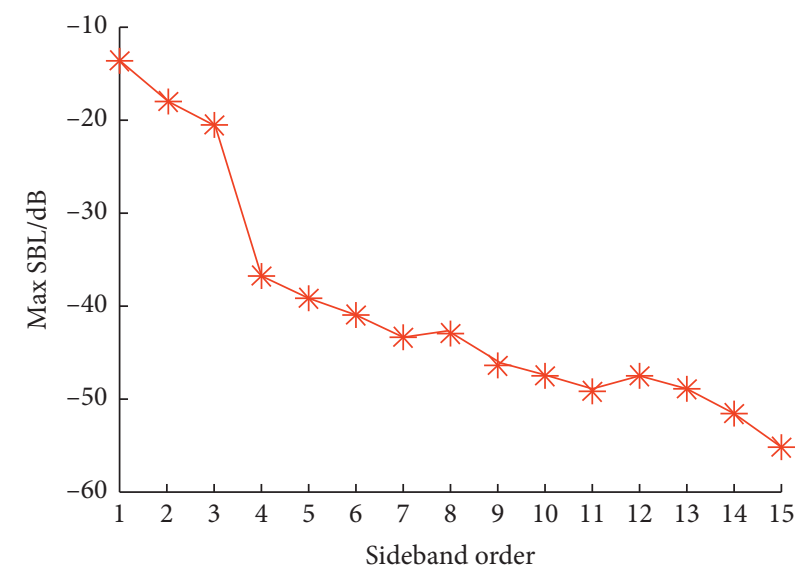

FIgURE 13: The SBL from the second sideband to the $20^{\text {th }}$ sideband.

the $15^{\text {th }}$-order sideband after optimization. Figure 13 shows the convergence curve of the second stage of the optimization.

The results of Figures 12 and 13 show that the ideal pattern is obtained by using the proposed method after adding the high-order sideband beamforming constraint. The maximum relative levels of the first three-order sideband beam direction with nonbeam direction (SLL1, SLL2, and SLL3) are $-19.73 \mathrm{~dB},-17.43 \mathrm{~dB}$, and $-18.02 \mathrm{~dB}$, and the sideband nonbeam direction meets the given constraints $u_{3}$. The results of Figure 14 show that the proposed algorithm converges after the eighth iteration for the convex optimization in the second stage. Compared with single order sideband optimization, more iterations are needed to achieve convergence.

It can be seen from the three figures that the SLL1 of the first sideband will be slightly reduced while achieving the effect of multiple sideband beam patterns due to the addition of high-order sideband constraints. At the same time, the number of times to the end of iteration and the time consumed by iteration will also increase. 


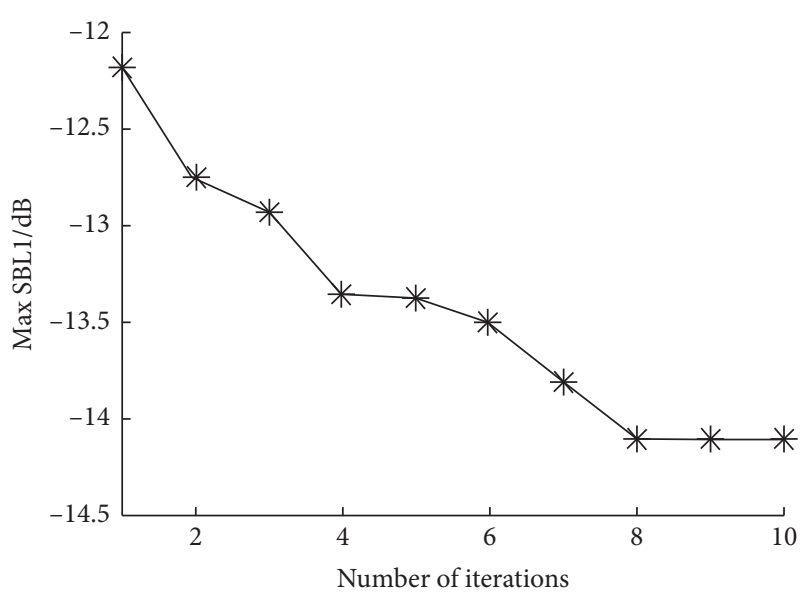

Figure 14: Convergence curve of the second stage.

\section{Conclusion}

A two-stage convex optimization method for sideband multibeamforming of TMA is proposed in this paper. The synthesis problem is divided into two parts: the first part uses the sequential convex optimization method to optimize the array static excitation and the switch-ON duration time to suppress the sidelobe of the center frequency. In the second part, the iterative convex optimization method is used to optimize the switch-ON time of the array elements and form the beam in the specified direction of the sideband. Simulation results show that the proposed method can effectively realize the single sideband and multisideband beamforming of time modulated array under the sidelobe of the main frequency band is effectively suppressed. In the case of DRR constraints and sideband radiation direction changes, the optimization performance of the algorithm is verified.

\section{Data Availability}

The data used to support the findings of this study are available from the corresponding author upon request.

\section{Conflicts of Interest}

The authors declare that there are no conflicts of interest regarding the publication of this paper.

\section{Acknowledgments}

This research was funded by the National Natural Science Foundation of China under (Grant no. 61671239) and the Fundamental Research Funds for the Central Universities (Grants nos. kfjj20200412 and kfjj20200417).

\section{References}

[1] H. E. Shanks and R. W. Bickmore, "Four-dimensional electromagnetic radiators," Canadian Journal of Physics, vol. 37, no. 3, pp. 263-275, 1959.

[2] S. Yang, Y. B. Gan, and P. K. Tan, "Evaluation of directivity and gain for time-modulated linear antenna arrays,"
Microwave and Optical Technology Letters, vol. 42, no. 2, pp. 167-171, 2004.

[3] Q. Zhu, S. Yang, L. Zheng, and Z. Nie, "Design of a low sidelobe time modulated linear array with uniform amplitude and sub-sectional optimized time steps," IEEE Transactions on Antennas and Propagation, vol. 60, no. 9, pp. 4436-4439, 2012.

[4] I. Kanbaz, U. Yesilyurt, S. Kuzu, and E. Aksoy, "Total harmonic power of arbitrarily switched nonuniform period time modulated arrays," IEEE Antennas and Wireless Propagation Letters, vol. 19, no. 1, pp. 193-197, 2019.

[5] C. He, H. Yu, X. Liang, J. Geng, and R. Jin, "Sideband radiation level suppression in time-modulated array by nonuniform period modulation," IEEE Antennas and Wireless Propagation Letters, vol. 14, pp. 606-609, 2015.

[6] E. Aksoy and E. Afacan, "An inequality for the calculation of relative maximum sideband level in time-modulated linear and planar arrays," IEEE Transactions on Antennas and Propagation, vol. 62, no. 6, pp. 3392-3397, 2014.

[7] T. Nakanishi, K. Kihira, T. Takahashi, Y. Konishi, and I. Chiba, "Sideband suppression using switched phase distribution in time-modulated array antennas," in Proceedings of the 2013 IEEE International Symposium on Phased Array Systems and Technology, Waltham, MA, USA, October 2013.

[8] M. Roberto, B. Mar, B. Julio, A. G. José, and C. Luis, "Beamsteering in switched $4 \mathrm{D}$ arrays based on the discrete walsh transform," in Proceedings of the 2019 27th European Signal Processing Conference (EUSIPCO), A Coruna, Spain, September 2019.

[9] O. Gassab, A. Azrar, A. Dahimene, S. Bouguerra, and C. He, "Efficient electronic beam steering method in time modulated linear arrays," IET Microwaves, Antennas \& Propagation, vol. 14, no. 5, pp. 402-408, 2020.

[10] H. Li, Y. Chen, and S. Yang, "Time-modulated beamforming in antenna arrays with multiple sub-branch RF switches," in Proceedings of the 2019 IEEE Asia-Pacific Microwave Conference (APMC), Singapore, December 2019.

[11] L. Poli, P. Rocca, and A. Massa, "Pulse sequence optimization for harmonic-diversity exploitation of time-modulated arrays in cognitive radio applications," in Proceedings of the 2018 IEEE International Symposium on Antennas and Propagation \& USNC/URSI National Radio Science Meeting, Boston, MA, USA, July 2018.

[12] M. Roberto, B. Julio, A. Jose, and C. Luis, "Time modulated array controlled by periodic pulsed signals," in Proceedings of the 2018 European Signal Processing Conference (EUSIPCO), Rome, Italy, September 2018.

[13] U. Yesilyurt, I. Kanbaz, S. Kuzu et al., "A non-iterative convoluted harmonic beamforming technique in time modulated arrays," IEEE Transactions on Antennas and Propagation, no. 99, p. 1, 2020.

[14] R. Maneiro-Catoira, J. C. Brégains, J. A. García-Naya, and L. Castedo, "Enhanced time-modulated arrays for harmonic beamforming," IEEE Journal of Selected Topics in Signal Processing, vol. 11, no. 2, pp. 259-270, 2017.

[15] S. K. Mandal, G. K. Mahanti, and R. S. Ghatak, "Synthesis of simultaneous multiple-harmonic-patterns in time-modulated linear antenna arrays," Progress in Electromagnetics Research $M$, vol. 34, pp. 135-142, 2014.

[16] Z. J. Jiang, S. Zhao, Y. Chen, and T. J. Cui, "Beamforming optimization for time-modulated circular-aperture grid array with DE algorithm," IEEE Antennas and Wireless Propagation Letters, vol. 17, no. 12, pp. 2434-2438, 2018.

[17] P. Rocca, Q. Zhu, E. T. Bekele, S. Yang, and A. Massa, "4-D arrays as enabling technology for cognitive Radio systems," 
IEEE Transactions on Antennas and Propagation, vol. 62, no. 3, pp. 1102-1116, 2014.

[18] Z. Zhang, H. E. Najafabadi, and L. Sun, "Shared aperture multibeam forming of time-modulated linear array," Complexity, vol. 2019, no. 4, 10 pages, Article ID 4101909, 2019.

[19] S. P. Boyd and L. Vandenberghe, Convex Optimization, Cambridge University Press, Cambridge, England, 2004.

[20] Y. Gong, S. Xiao, and B. Wang, "Synthesis of nonuniformly spaced arrays with frequency-invariant shaped patterns by sequential convex optimization," IEEE Antennas and Wireless Propagation Letters, vol. 19, no. 7, pp. 1093-1097, 2020.

[21] M. D’Urso, G. Prisco, and R. M. Tumolo, “Maximally sparse, steerable, and nonsuper directive array antennas via convex optimizations," IEEE Transactions on Antennas and Propagation, vol. 64, no. 9, pp. 3840-3849, 2016.

[22] Z. X. Huang and Y. J. Cheng, "Near-field pattern synthesis for sparse focusing antenna arrays based on Bayesian compressive sensing and convex optimization," IEEE Transactions on Antennas and Propagation, vol. 66, no. 10, pp. 5249-5257, 2018.

[23] L. Poli, P. Rocca, L. Manica, and A. Massa, "Pattern synthesis in time-modulated linear arrays through pulse shifting," IET Microwaves, Antennas \& Propagation, vol. 4, no. 9, pp. 1157-1170, 2010.

[24] Q. Zhu, S. Yang, R. Yao, M. Huang, and Z. Nie, "Unified timeand frequency-domain study on time-modulated arrays," IEEE Transactions on Antennas and Propagation, vol. 61, no. 6, pp. 3069-3076, 2013.

[25] F. Yang, S. Yang, Y. Chen, S. Qu, and J. Hu, "Convex optimization of pencil beams through large-scale 4-D antenna arrays," IEEE Transactions on Antennas and Propagation, vol. 66, no. 7, pp. 3453-3462, 2018.

[26] J. Gorski, F. Pfeuffer, and K. Klamroth, "Biconvex sets and optimization with biconvex functions: a survey and extensions," Mathematical Methods of Operations Research, vol. 66, no. 3, pp. 373-407, 2007.

[27] P. You, Y. Liu, S.-L. Chen, K. Da Xu, W. Li, and Q. H. Liu, "Synthesis of unequally spaced linear antenna arrays with minimum element spacing constraint by alternating convex optimization," IEEE Antennas and Wireless Propagation Letters, vol. 16, pp. 3126-3130, 2017.

[28] F. Yang, S. Yang, Y. Chen, S. Qu, and J. Hu, "Efficient pencil beam synthesis in 4-D antenna arrays using an iterative convex optimization algorithm," IEEE Transactions on Antennas and Propagation, vol. 67, no. 11, pp. 6847-6858, 2019.

[29] E. Aksoy, "Calculation of sideband radiations in time-modulated volumetric arrays and generalization of the power equation," IEEE Transactions on Antennas and Propagation, vol. 62, no. 9, pp. 4856-4860, 2014. 